\title{
Curso de prevenção ao uso de drogas: Descrição e avaliação de satisfação
}

Érika Pizziolo Monteiro. Universidade Federal de Juiz de Fora. Henrique Pinto Gomide. Centro de Ensino Superior de Juiz de Fora. Pollyanna Santos da Silveira. Universidade Católica de Petrópolis. Telmo Mota Ronzani. Universidade Federal de Juiz de Fora.

\section{Resumo}

Diante do cenário de uso de substâncias por crianças e adolescentes, é relevante implementar ações de prevenção no ambiente escolar. Dentre elas, cita-se a utilização de cursos online para capacitar os diversos atores que podem ser protagonistas das atividades propostas. O presente estudo apresentou e avaliou o Curso de Prevenção do Uso de Drogas para Educadores de Escolas Públicas. Participaram 4.960 educadores de Minas Gerais, Rio de Janeiro e Paraná e foram investigadas as características sociodemográficas destes, além da avaliação de aspectos de satisfação com o curso e sobre o acesso às ferramentas virtuais de aprendizagem. O perfil dos participantes foi composto predominantemente por mulheres $(85,8 \%)$ com pós-graduação $(64,8 \%)$ e 35,2\% afirmaram possuir contato com a temática álcool e drogas no contexto escolar. A maioria dos participantes aprovou o curso $(98,1 \%)$, indicando que o método de ensino e estratégias utilizados se mostraram adequados ao público capacitado.

Palavras-chave: prevenção do abuso de drogas; educação à distância; avaliação de programa.

\section{Abstract}

Drug abuse prevention course: Description and evaluation of satisfaction. Considering the substance use by children and adolescents, it is important to implement preventive actions in the school environment. Among them, there is the use of online courses to train the various actors who can be protagonists of the planed activities. This study presented and evaluated the Drug Use Prevention Course for Public School Educators. A total of 4,960 educators from Minas Gerais, Rio de Janeiro, and Paraná participated and the sociodemographic characteristics of these, in addition to evaluation of aspects of satisfaction with the course and access to virtual learning tools were investigated. The profile of the participants was predominantly composed of women (85.8\%) with a postgraduate degree $(64.8 \%)$ and $35.2 \%$ reported having contact with the alcohol and drugs theme in the school context. Most participants approved the course (98.1\%), indicating that the teaching method and strategies used were suitable to the qualified public.

Keywords: drug abuse prevention; distance education; program evaluation.

\section{Resumen}

Curso de prevención del consumo de drogas: Descripción y evaluación de satisfacción. En el contexto del uso de sustancias por niños y adolescentes, es importante implementar acciones preventivas en el ámbito escolar. En este estudio se presentó y evaluó el Curso de Prevención del uso de drogas a Educadores de Escuelas Públicas. En total participaron 4960 educadores de Minas Gerais, Río de Janeiro y Paraná. La investigación se centró en: las características sociodemográficas de estos, la evaluación y el nivel de satisfacción con el curso; y el acceso a las herramientas virtuales de aprendizaje. El perfil de los participantes estaba compuesto predominantemente por mujeres $(85,8 \%)$ con un título de postgrado $(64,8 \%)$. El 35,2\% informó "haber desarrollado el tema de alcohol y otras drogas en el contexto escolar. La mayoría de los participantes aprobaron el curso (98,1\%), lo que indica que el método de enseñanza y las estrategias utilizadas fueron adecuados para el público especializado.

Palabras clave: prevención en el abuso de drogas; educación a distancia; evaluación de programa. 
Vários fatores podem influenciar os padrões de consumo de bebidas alcoólicas, alguns desses fatores são individuais e outros, ambientais. A Organização Mundial da Saúde (OMS) aponta, em um levantamento publicado em 2014, a idade como um destes determinantes. Segundo esse levantamento, na região das Américas, $29 \%$ dos adolescentes entre 15 e 19 anos são abstinentes, enquanto $18,3 \%$ costumavam beber e $52,7 \%$ bebem atualmente (OMS, 2014). Um relatório produzido pela Organização Panamericana de Saúde (OPAS) discorre sobre os episódios de consumo excessivo de álcool, apontando que, no Brasil, $21,2 \%$ das meninas consumidoras de álcool entre 15 e 19 anos relataram consumo excessivo, enquanto $62 \%$ dos meninos nesta condição reportaram tal padrão de consumo (OPAS, 2015).

O VI Levantamento Nacional sobre o Consumo de Drogas Psicotrópicas entre Estudantes do Ensino Fundamental e Médio das Redes Pública e Privada de Ensino nas 27 Capitais Brasileiras (Brasil, 2010) aponta que bebidas alcoólicas e o tabaco têm sido as substâncias mais consumidas pelos adolescentes $(60,5 \%$ e $16,9 \%$, respectivamente, relataram uso na vida). E apesar da grande maioria dos estudantes afirmarem nunca ter feito uso de qualquer substância ilegal, ao longo dos anos observou-se um crescente relato do consumo de maconha e cocaína no Brasil (Brasil, 2010). Diante deste cenário, a Política Nacional sobre Drogas (PNAD) propõe diferentes estratégias para reduzir o impacto do uso de álcool e drogas à saúde. Entre essas medidas, encontram-se as ações de prevenção, as ações de promoção e proteção de consumidores, os serviços de tratamento e os de controle de entorpecentes (Brasil, 2003b; Brasil, 2005).

Com o aumento do número das ações de prevenção, a demanda pela avaliação dessas ações foi acentuada, assim como a difusão dos princípios preventivos cientificamente efetivos (Champion, Newton, Barrett, \& Teesson, 2013). Para que as ações de prevenção sejam realizadas de forma mais eficaz, recomenda-se que os profissionais envolvidos nas atividades sejam sensibilizados sobre a temática álcool e outras drogas. Dentre essas iniciativas estão os cursos de capacitação à distância que buscam preparar profissionais para lidar com usuários de álcool e outras drogas. A proposta envolve um número significativo de profissionais de diversas áreas e cada curso possui seus respectivos objetivos e metodologias (Carneiro, 2014).

$\mathrm{Na}$ literatura, existem diversos exemplos de processos avaliativos de ações destinadas à prevenção do uso abusivo de álcool e outras drogas. Um estudo realizado pelo United Nations Office for Drug Control and Crime Prevention's (UNODCCP, 2002) evidencia a importância da identificação de ações que envolvam estratégias preventivas eficientes, apontando os componentes eficazes, a relação custo-benefício e a probabilidade de adaptação do programa aos contextos em que as ações de prevenção são operacionalizadas (Winters, Fawkes, Fahnhorst, Botzet, \& August, 2007).

A educação à distância se caracteriza como uma alternativa economicamente viável para a capacitação de um grande número de profissionais, entre eles educadores públicos. O processo ensino-aprendizagem em uma perspectiva de educação continuada permite que novos temas sejam abordados pelos profissionais em suas práticas diárias. Entretanto, para que este processo seja produtivo, características específicas dos atores envolvidos são requisitos para a obtenção de bons resultados, como por exemplo, disciplina por parte do profissional que está sendo capacitado para realizar as tarefas propostas pelo curso e uma dinâmica de construção de conhecimento clara e embasada no que se refere à organização do processo de capacitação (Emanuelli, 2011; Garcia \& Baptista, 2007; Oliveira, 2007).

Diante da necessidade de realizar ações que tragam resultados positivos no sentido de prevenir o uso abusivo de álcool e outras drogas, ressalta-se a importância de uma cultura de avaliação das ações propostas e realizadas, devido ao fato do processo de avaliação e os resultados a este associados serem usados na melhoria de aspectos que podem não ter produzido o efeito inicialmente esperado (OMS, 2002). Com relação aos cursos à distância, a necessidade de avaliação se relaciona aos poucos estudos na área quando comparado ao aumento considerável do uso desta modalidade de ensino como processo formativo de profissionais (Abbad, Zerbini, \& Souza, 2010).

Considerando que a avaliação de uma proposta implica em coleta e análises sistemáticas de informações sobre esta, divulgando-as para uso no processo de tomada de decisões (Rush \& Nadeau, 2011), a investigação das questões relacionadas ao desenvolvimento, implementação e avaliação das ações direcionadas à prevenção do uso abusivo de álcool e outras drogas se mostra necessária para que práticas eficazes sejam desenvolvidas e divulgadas para a comunidade científica e diversas equipes de profissionais interessadas em trabalhar a temática.

Sendo assim, o presente artigo objetiva realizar uma avaliação do Curso de Prevenção do Uso de Drogas para Educadores de Escolas Públicas, através de 
indicadores de caracterização dos participantes, tipo de acesso ao Ambiente Virtual de Aprendizagem no qual o processo foi operacionalizado e satisfação com o curso.

\section{Métodos}

Apresentação do "Curso de Prevenção do Uso de Drogas para Educadores de Escolas Públicas

Dentre os diversos cursos à distância oferecidos no contexto brasileiro, propostos e executados pelo Governo Federal, encontra-se o "Curso de Prevenção do Uso de Drogas para Educadores de Escolas Públicas" cujo objetivo é promover a formação de profissionais das escolas públicas para atuarem coletivamente na prevenção do uso álcool e outras drogas no contexto escolar (Brasil, 2012). O curso foi promovido pela Secretaria Nacional de Políticas sobre Drogas (SENAD), vinculada ao Ministério da Justiça (MJ), juntamente com a Secretaria de Educação Básica do Ministério da Educação (MEC).

O curso foi realizado entre os meses de abril e dezembro de 2014, totalizando 8 meses de duração. 0 curso se inseriu na modalidade Educação à Distância, sendo disponibilizado através da Plataforma Moodle. Ele foi composto por cinco módulos, sendo os quatro primeiros teóricos e o último voltado para a implementação das ações planejadas durante os módulos anteriores. Cada um dos módulos foi composto por unidades que apresentavam objetivos específicos. Estes objetivos abordavam aspectos como o reconhecimento da escola enquanto espaço de promoção de saúde, sendo considerada como um ambiente de desenvolvimento de ações de prevenção baseadas nas diretrizes de políticas públicas, utilizando-se do potencial das redes sociais como metodologia de prevenção. Por fim, as ações de prevenção propostas, seguindo as diretrizes pontuadas pelo material didático do curso, reconhecem a importância da formação continuada do educador, considerando as demandas sociais relativas à promoção da saúde e prevenção do uso de álcool e outras drogas.

Os aspectos propostos como efetivos pela literatura científica para a construção de um programa de prevenção foram abordados no Curso de Prevenção do Uso de Drogas para Educadores de Escolas Públicas (Barbosa, Pereira, \& Oliveira, 2014; Canoletti \& Soares, 2005). As atividades apresentadas aos cursistas foram formuladas com base no conteúdo do material didático específico do curso, confeccionado e distribuído pela Universidade de Brasília (Brasil, 2012).
Estratégias pedagógicas foram utilizadas a fim de maximizar o processo de ensino-aprendizagem, buscando o envolvimento dos educadores-cursistas na realização das tarefas propostas, essenciais para a construção do projeto de prevenção ao uso abusivo de álcool e outras drogas. Tais estratégias foram:

1. Quinze Fóruns Virtuais de Discussão do conteúdo específico de cada unidade. Cada Fórum objetivava criar espaço para discussão de uma questão formulada pelo professor do Módulo, abordando os objetivos da unidade.

2. Atividades Colaborativas realizadas por educadores pertencentes ao mesmo grupo escolar ao final de cada módulo (quatro ao todo). Cada uma das atividades objetivou a construção de uma etapa do projeto de prevenção ao uso de álcool e outras drogas a ser implementado no contexto escolar, objetivo primário do curso.

3. Questionário avaliativo e conteudista ao final de cada Módulo - respondido por todos educadores. Essa atividade foi realizada três vezes, não sendo aplicada durante o módulo 4 em virtude do maior número de Fóruns de Discussão desta etapa. Estes questionários abordavam questões teóricas chave sobre as temáticas apresentadas no material didático, buscando avaliar o entendimento dos cursistas de pontos essenciais sobre o processo de prevenção do uso de álcool e outras drogas, discutidos em cada Módulo.

A equipe responsável pelo desenvolvimento do curso envolveu profissionais do Centro de Educação à Distância da Universidade, responsáveis pela elaboração das mídias a serem utilizadas, além da construção do Ambiente Virtual de Aprendizagem e profissionais/ pesquisadores da área de álcool e outras drogas, que compuseram a equipe pedagógica do curso.

Uma vez estabelecidas as atividades que comporiam o quadro de notas do curso, definiu-se que seriam aprovados os cursistas que obtivessem um mínimo de $60 \%$ das notas totais distribuídas entre as atividades. Durante cada Módulo, um total de 100 pontos foi distribuído entre as questões dos Fóruns de Discussão, a Atividade Colaborativa e o Questionário Conteudista, atribuindo-se sempre um maior peso ao produto da atividade colaborativa, visto a importância do desenvolvimento dos projetos de prevenção - objetivo destas atividades, conforme citado anteriormente. A mediação das atividades era feita pelos tutores que atribuíam as notas com base em critérios estabelecidos pelos professores dos Módulos e discutidos entre tutores e coordenação de tutoria. Este processo de aprovação 

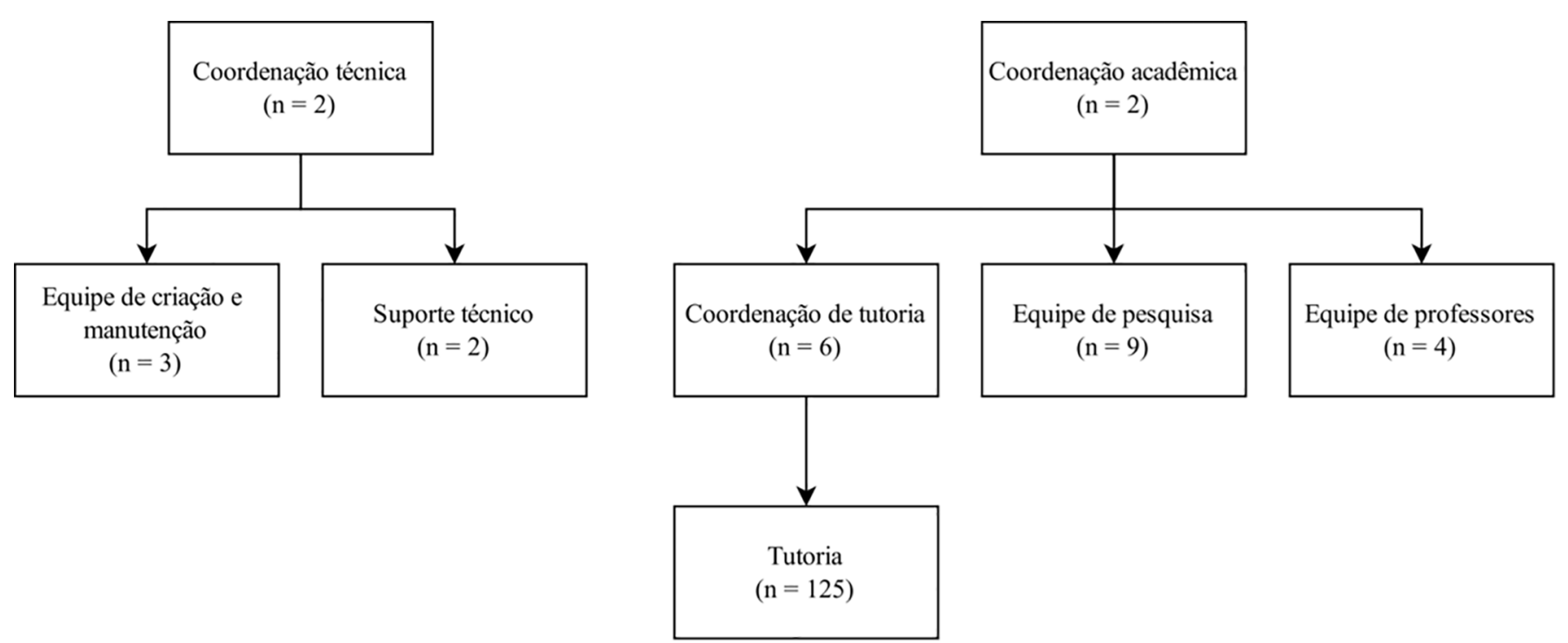

Figura 1. Fluxograma de Organização da Equipe do Curso de Prevenção do Uso de Drogas para Educadores de Escolas Públicas.

ocorreu de duas formas: através de participação via Ambiente Virtual de Aprendizagem (AVA) e via processo de recuperação, realizado também de forma virtual, porém, em um dispositivo disponibilizado pelo Centro de Educação à Distância (CEAD-UFJF) para que questões chave sobre temáticas do curso fossem discutidas. Para o presente estudo, apenas os participantes das atividades realizadas no AVA foram considerados.

\section{Participantes}

Foram inscritos 4960 educadores de escolas públicas dos estados de Minas Gerais, Rio de Janeiro e Paraná. Devido ao fato de o curso ter uma duração considerável, as coletas das informações necessárias aos objetivos almejados no presente artigo foram feitas em momentos distintos. Sendo assim, foram realizadas duas coletas de dados para obter os resultados pretendidos. Inicialmente foram coletadas informações sociodemográficas tais como sexo, idade, escolaridade, ocupação e contato prévio com a temática álcool e outras drogas (obtidas ao final do Módulo 1 do curso) $(n=3.247)$ e, posteriormente, as informações de avaliação de aspectos do curso e sobre o acesso às ferramentas virtuais de aprendizagem (coletadas ao final do Módulo 4) $(n=1.950)$.

\section{Instrumentos}

No presente estudo, foram investigadas as variáveis abaixo listadas através de questionários de caracterização dos participantes do curso e dos aspectos organizacionais desse, disponibilizados virtualmente.
1. Questionário sócio-demográfico - aplicado ao final do módulo 1 com o objetivo de caracterizar a amostra a partir das seguintes variáveis: sexo, idade, nível de formação, ocupação, contato prévio com a temática álcool e outras drogas, motivos para participação do curso.

2. Questionário de avaliação dos processos pedagógicos - aplicado ao final do módulo 4 com o objetivo de avaliar o curso a partir das seguintes questões: adequação do material didático, flexibilidade do prazo de entrega das atividades, avaliação da importância das atividades nos fóruns - interação com outros cursistas, organização do curso, importância do auxílio do tutor. Este questionário foi usado para fins de pesquisa, não sendo, portanto, atribuído a ele notas. As informações nele contidas foram desenvolvidas e analisadas pela equipe de pesquisa do Curso de Prevenção do Uso de Drogas para Educadores de Escolas Públicas.

3. Registros de atividades - participação dos alunos extraída ao final do curso usando os registros de acessos da plataforma virtual.

\section{Procedimentos}

Coleta de Dados. A coleta de dados foi realizada de maneira virtual, através de questionários com questões fechadas para as variáveis sociodemográficas e avaliativas do curso. Os registros de acesso à plataforma foram utilizados para coletar as variáveis de realização das atividades pedagógicas. Os dados foram extraídos utilizando os módulos de questionário oferecidos pela plataforma Moodle. 
Análise dos dados. Para caracterização da amostra foram utilizadas estatísticas descritivas como frequências, médias e desvios-padrão e métodos gráficos. As análises foram conduzidas na linguagem R (R Core Team, 2015) usando os seguintes pacotes ggplot2 (Wickham, 2009) e plyr (Wickham, 2011). Para facilitar a replicabilidade da pesquisa, os códigos usados para extração e análise como também os bancos de dados com identificações protegidas foram disponibilizados no repositório http:// github.com/ead-senad/preditores.

\section{Considerações Éticas}

O processo metodológico neste artigo descrito foi vinculado a um projeto de maior abrangência denominado "Capacitação e Avaliação de Educadores de Escolas Públicas para Prevenção do Uso de Drogas entre Adolescentes", aprovado pelo Comitê de Ética em Pesquisa da Universidade Federal de Juiz de Fora (UFJF) através do parecer de número 787.766. Foram incluídos no presente estudo, os cursistas que voluntariamente concordaram com o Termo de Consentimento Livre e Esclarecido e responderam aos questionários disponibilizados no decorrer do curso.

\section{Resultados}

Do total de 4.690 educadores-cursistas, 3.247 (69\%) participaram do primeiro momento de coleta. A maioria dos respondentes eram mulheres $(85,8 \%)$ e a média de idade foi 40,7 anos (DP: $\pm 8,95$ ). A Tabela 1 apresenta os resultados referentes à escolaridade, ocupação e contato anterior com a temática álcool e outras drogas dos participantes.

O questionário com questões relacionadas à motivação e satisfação de participação no curso foi respondido virtualmente por 1950 educadores-cursistas (41,56\% dos alunos ativos durante o período de coleta de dados) durante o período de finalização das atividades do Módulo 4, período este em que 2.429 alunos se encontravam ativos no curso.

Com relação às razões para participação no curso, 29,1\% responderam "por ser uma oportunidade de formação continuada", 17,2\% "por identificação profissional com o tema", 3,1\% "por identificação pessoal com o tema", $0,7 \%$ "pelo fato de o curso estar vinculado à Universidade", 0,4\% "Por ser um curso à distância" e $0,2 \%$ "pelo fato de o curso ser gratuito".

Em relação ao material didático, 99,3\% dos participantes consideraram-no muito adequado ou
Tabela 1. Informações referentes a escolaridade, ocupação e contato anterior com a temática álcool e outras drogas sobre os participantes do Curso de Prevenção do Uso de Drogas para Educadores de Escolas Públicas.

\begin{tabular}{lc}
\hline Escolaridade & \\
\hline Pós-Graduação & $64,8 \%$ \\
Ensino Superior (Completo ou Incompleto) & $33,8 \%$ \\
Ensino Médio & $1,3 \%$ \\
\hline Ocupação & $70,9 \%$ \\
\hline Professor(a) & $5,2 \%$ \\
Diretor (a) & $4,4 \%$ \\
Coordenador (a) Pedagógico (a) & $19,5 \%$ \\
\hline Outros & $35,2 \%$ \\
\hline Contato anterior com a temática álcool e outras drogas (Sim $=62,8 \%)$ \\
\hline Contato no contexto escolar (Sim) & $22,8 \%$ \\
Familiares usuários ou dependentes (Sim) & $18,6 \%$ \\
\hline
\end{tabular}

adequado e $0,7 \%$ como pouco adequado ou nada adequado. Sobre a flexibilidade dos prazos para realização das atividades, $65,3 \%$ avaliaram este como "flexível", 17,4\% como "muito flexível", 17,3\% como "pouco ou nada flexível".

Sobre a importância de interação com outros colegas através do ambiente virtual de aprendizagem, $50,3 \%$ dos respondentes avaliaram tal interação como "muito importante", 45,1\% como "importante" e 4, 7\% como "pouco ou nada importante". Com relação à organização do curso de uma forma geral, $50,6 \%$ consideraram o curso "organizado", 47,5\%, "muito organizado" e 1,9\% "desorganizado ou muito desorganizado". Sobre a mediação e auxílio do tutor à distância, 75,1\% consideram tal auxílio como "sempre importante", 22,3\% disseram que este suporte é necessário "às vezes" e 2,7\% consideraram que tal auxílio "não é necessário".

No que se refere à realização das atividades propostas, encontram-se abaixo os gráficos que ilustram a participação nos Fóruns de Discussão, realização das Atividades Colaborativas e preenchimentos dos Questionários Avaliativos. Ressalta-se que devido ao fato de haver cinco Fóruns de Discussão durante o Módulo 4, neste Módulo excepcionalmente não houve aplicação de Questionário Avaliativo.

\section{Discussão}

O presente trabalho buscou cobrir a lacuna de estudos descritivos sobre cursos de educação à 

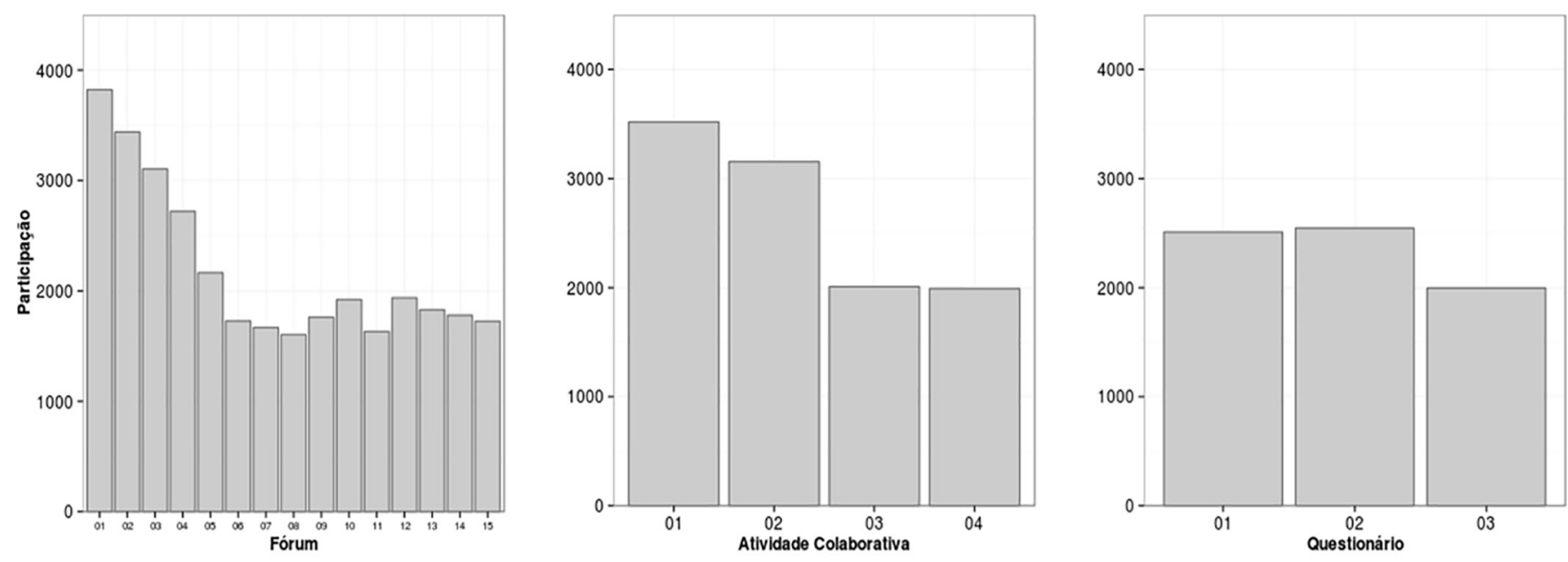

Figura 2. Gráficos de Barras da Participação dos Cursistas Separadas por Fóruns, Atividades Colaborativas e Questionários.

distância com a temática álcool e outras drogas no contexto brasileiro. Embora existam livros e trabalhos que descrevam tais cursos (Abbad et al., 2010), poucos são aqueles que concentram-se na descrição dos participantes e na avaliação da satisfação e no uso das atividades como um processo avaliativo do curso oferecido (Schweitzer \& Rodrigues, 2013).

Diante do reconhecimento da necessidade de avaliação dos processos de formação continuada, estabeleceu-se como primeira etapa deste estudo, a identificação do perfil dos cursistas. O perfil dos participantes do presente estudo foi composto predominantemente por mulheres professoras com pós-graduação. Entretanto, coordenadores pedagógicos e diretores também estavam representados. Identificar as características dos cursistas de iniciativas de educação à distância torna-se importante uma vez que em edições posteriores dos cursos, tais informações podem ser consideradas a fim de planejar conteúdos, propostas de discussões e atividades, tornando-as adequadas aos profissionais que irão aplicar os conhecimentos adquiridos. Neste sentido, identificar, no presente estudo, que diversos profissionais de comunidades escolares estão envolvidos em propostas de prevenção do uso abusivo de álcool e outras drogas apresenta-se como uma informação potencial à implementação de projetos futuros, uma vez que vários aspectos podem ser abordados pelos diversos atores atuantes na realização das atividades de prevenção (Rohrbach, Graham, \& Hansen, 1993).

No que se refere à discussão sobre a temática álcool e outras drogas, a maioria dos cursistas afirmou possuírem contato com a temática. Desses, a maior parte afirmou que o contato ocorreu no próprio contexto escolar. Esse dado indica a relevância do tema e da necessidade de projetos específicos serem trabalhados na própria escola, de forma contínua e presente nas atividades realizadas em sala de aula (Barbosa et al., 2014). Projetos de prevenção ao uso de álcool e outras drogas devem ser planejados e executados com base em indicações científicas, de forma a fortalecer as possibilidades de sucesso ao se buscar discutir e intervir em uma questão social complexa, como o fenômeno do abuso de álcool e outras drogas. Usar estudos consistentes no desenvolvimento de propostas de formação continuada de profissionais apresenta-se como estratégia de construção de práticas mais eficazes.

Sobre a satisfação dos educadores, de uma forma geral, os cursistas se posicionaram de forma bastante positiva em relação ao curso, indicando que o método de ensino e estratégias utilizados se mostraram adequados ao público capacitado pelo Curso de Prevenção do Uso de Drogas para Educadores de Escolas Públicas. Entretanto, a perda de alunos durante o período do curso foi substancial. Dos 10.000 alunos inscritos, 2.180 foram certificados pela participação via Plataforma Moodle, representando $21,8 \%$ dos alunos inscritos. A perda de alunos em cursos à distância é um fenômeno conhecido e estudado, a literatura existente na área aponta que a desistência de participação nos cursos oferecidos em modalidade à distância é significativa, identificando os motivos que justificam tal fenômeno. Conceitos como adesão ao curso oferecido e permanência no processo, seja este de graduação ou de formação continuada, também são discutidos (Abbad, Carvalho, \& Zerbini, 2006; Fiuza \& Sarriera, 2013). 
Considerando o cenário de ampla utilização das metodologias de ensino à distância, avaliar se tal postura é seguida por bons resultados quanto ao processo de construção do conhecimento faz-se necessário, uma vez que investimentos em políticas públicas devem ser avaliados para estudo sobre a eficácia dos métodos utilizados (Mota, Costa, Ronzani, Colugnati, \& Rush, 2014).

Rush (2003) propõe componentes que devem ser analisados em um processo de avaliação de programas na área de álcool e outras drogas. Dentre estes componentes estão: avaliação de necessidades, planejamento da avaliação, avaliação de processo, avaliação de custos, satisfação de usuários, avaliação de resultados e avaliação econômica. O presente artigo enfatiza determinadas áreas de avaliação e parte do pressuposto de que o processo de avaliação é essencial para que decisões quanto à replicação das práticas de prevenção em álcool e outras drogas sejam tomadas. Nesse sentido, no contexto brasileiro, fazse necessário entender como as ações preparatórias para o desenvolvimento de práticas de prevenção ocorrem, descrevendo-as em termos de seus objetivos e metodologias e discutindo se os resultados provenientes destas são satisfatórios.

Considerando os resultados encontrados, concluise que as estratégias pedagógicas adotadas foram suficientes para atingir ao objetivo de sensibilizar os educadores que passaram pelo processo de capacitação quanto à relevância da temática de prevenção ao uso de abusivo de álcool e outras drogas, trazendo tal discussão aos contextos escolares e permitindo que ações sejam planejadas e implementadas de forma eficiente e com base na literatura existente na área. Entretanto, uma vez que os resultados apresentados são referentes a participantes que continuaram a realizar o curso até a fase final, ressalta-se que aspectos como a satisfação com o processo formativo são esperados a serem avaliados de forma positiva, fazendo-se necessário investigar, em futuras propostas, os motivos pelos quais educadores iniciaram o curso e não o concluíram.

Diante do cenário acima apresentado, abordar a questão da educação à distância enquanto estratégia para propiciar o processo de desenvolvimento e implementação de projetos de prevenção do uso abusivo de álcool e outras drogas enquanto execução das políticas públicas existentes na área de drogas no contexto brasileiro se faz necessário. Dessa forma, considerando a perspectiva de avaliação das ações de prevenção ao uso abusivo de álcool e outras drogas, entre elas, a importância da avaliação das iniciativas de formação dos profissionais que lidam com a questão em suas práticas diárias, também é importante considerar a avaliação do impacto deste processo de capacitação e o consequente desenvolvimento de projetos de prevenção ao uso abusivo de álcool e outras drogas nas escolas, buscando informações sobre a implementação de tais projetos e sobre a existência de indicadores positivos como, por exemplo, o aumento da primeira idade do uso de substâncias por adolescentes ou a diminuição das taxas de consumo, conforme objetivo traçado pelo projeto. Tais informações também podem ser formas de avaliar a aquisição de conhecimento teórico e prático por parte dos educadores e o sucesso do processo de capacitação.

O presente estudo foi realizado com apoio da Secretaria Nacional de Políticas sobre Drogas (Senad), Ministério da Educação (MEC) e Conselho Nacional de Desenvolvimento Científico e Tecnológico (CNPq), órgãos essenciais para a realização das ações relacionadas ao Curso de Prevenção do Uso de Drogas para Educadores de Escolas Públicas.

\section{Conclusão}

O objetivo deste estudo é identificar as características do público-alvo de um curso de sensibilização para a construção de práticas preventivas em contextos escolares na área de álcool e outras drogas. Diante deste contexto e, uma vez estabelecida a importância de desenvolver e implementar ações de caráter preventivo do uso abusivo de álcool e outras drogas, esforços para capacitar os profissionais designados a lidar com a temática devem ser priorizados de forma que o planejamento e execução de projetos com índices de efetividade comprovados possam ser adaptados à diversas realidades de contextos escolares. A Educação à Distância se apresenta como alternativa de formação continuada para disseminação de práticas preventivas entre educadores da rede pública de ensino brasileira, maximizando o alcance dessas. Como o presente trabalho, buscou-se enfatizar a relevância da questão do uso abusivo de álcool e outras drogas por crianças e adolescente e a importância de produzir práticas adequadas para intervenção sobre o fenômeno.

\section{Referências}

Abbad, G., Carvalho, R. S., \& Zerbini, T. (2006). Evasão em curso via internet: explorando variáveis explicativas. RAE-eletrônica, 5(2), 00-00. doi: 10.1590/S1676-56482006000200008 
Abbad, G. S., Zerbini T., \& Souza D. B. L. (2010). Panorama das pesquisas em educação a distância no Brasil. Estudos de Psicologia, 15(3), 291-298. doi: 10.1590/S1413-294X2010000300009

Barbosa, A.J. G., Pereira, C.E. S., \& Oliveira, J. C. (2014). Prevenção escolar ao uso de drogas por adolescentes: intervenções que funcionam. In T. M. Ronzani \& P. S. Silveira (Orgs.), Prevenção ao uso de álcool e outras drogas no contexto escolar (pp. 49-70). Juiz de Fora: UFJF.

Brasil, Ministério da Saúde (2003). A política do Ministério da Saúde para a atenção integral a usuários de álcool e outras drogas. Brasília, DF.

Brasil, Conselho Nacional Antidrogas (2005). Conselho Nacional Antidrogas. Política Nacional sobre Drogas. Brasília, DF.

Brasil, Secretaria Nacional de Políticas sobre Drogas (SENAD) (2010). VI Levantamento Nacional sobre o Consumo de Drogas Psicotrópicas entre Estudantes do Ensino Fundamental e Médio das Redes Pública e Privada de Ensino nas 27 Capitais Brasileiras. Brasília, DF.

Brasil, Secretaria Nacional de Políticas Sobre Drogas (SENAD) (2012). Curso de Prevenção do Uso de Drogas para Educadores de Escolas Públicas. Brasília: UnB.

Canoletti, B., \& Soares, C. B. (2005). Programas de prevenção ao consumo de drogas no Brasil: uma análise da produção científica de 1991 a 2001. Interface - Comunicação, Saúde, Educação, 9(16), 115-129. doi: 10.1590/S1414-32832005000100010

Carneiro, A. P. L. (2014). Efetividade de cursos à distância na capacitação de profissionais de saúde, assistência social e líderes religiosos/ comunitários para realizar triagem dos uso de drogas e intervenções breves (Dissertação de Mestrado não publicada). Universidade Federal de São Paulo, São Paulo, SP.

Champion, K. E., Newton, N. C., Barrett, E. L., \& Teesson, M. (2013). A systematic review of school-based alcohol and other drug prevention programs facilitated by computers or the internet. Drug and Alcohol Review, 32(2), 115-123. doi: 10.1111/j.1465-3362.2012.00517.x

Emanuelli, G. B. (2011). Atração e refração na educação a distância: constatações sobre o isolacionismo e a evasão do aluno. Revista Gestão Universitária na América Latina, 4(2), 205-218.

Fiuza, P. J., \& Sarriera, C. R. (2013). Motivos para adesão e permanência discente na educação superior a distância. Psicologia: Ciência e Profissão, 33(4), 884-901. doi: 10.1590/S1414-98932013000400009

Garcia, R. M., \& Baptista, R. (2007). Educação à distância para a qualificação dos profissionais do SUS: perspectivas e desafios. Revista Baiana de Saúde Pública, 31(Suppl. 1), 70-78.

Mota, D. C. B, Costa, P. H. A., Ronzani, T. M., Colugnati, F. A. B., \& Rush, B. R. (2014). Avaliação dos sistemas de tratamento para usuários de drogas no Brasil. In Ronzani T. M. (Org.), Intervenções e inovações em álcool e outras drogas (pp. 35-54). Juiz de Fora: UFJF.
Oliveira, M. A. N. (2007). Educação à distância como estratégia para a educação permanente em saúde: possibilidades e desafios. Revista Brasileira de Enfermagem, 60(5), 585-589. doi: 10.1590/S003471672007000500019

Organização Mundial de Saúde. (2002). Prevention of psychoactive substance use. A selected review of what works in the area of prevention. Geneva: WHO Press

Organização Mundial de Saúde. (2014). Global Status report on alcohol and health 2014. Geneva: WHO Press

Organização Panamericana de Saúde. (2015). Informe sobre la situación regional sobre el alcohol y la salud en las Américas. Washington, DC: OPAS

R Core Team. (2015). R: a language and environment for statistical computing. $R$ Foundation for Statistical Computing. Vienna: the $\mathrm{R}$ Project for Statistical Computing. Recuperado de http://www.Rproject.org/.

Rohrbach, L. A., Graham, J. W., \& Hansen, W. B. (1993). Diffusion of a school-based substance abuse prevention program: predictors of program implementation. Preventive Medicine 22(2), 237-260. doi: 10.1006/pmed.1993.1020

Rush, B. (2003). The evaluation of treatment services and systems for substance use disorders. Revista de Psiquiatria, 25(3), 393-411. doi: 10.1590/S0101-81082003000300002

Rush, B. R., \& Nadeau, L. (2011). Integrated service and system planning debate. In D. Cooper (Org.), Responding in mental health. Oxford: Radcliffe Publishing.

Schweitzer, F., \& Rodrigues, R. S. (2013). Produção científica em áreas multidisciplinares: educação a distância no Brasil. Revista da Faculdade de Biblioteconomia e Comunicação da UFRGS, 19(1), 156-172. doi: 10.19132/1808-5245191.156-172

United Nations Office for Drug Control and Crime Prevention (2002). Lessons learned in drug abuse prevention: a global review. Nova Iorque, NY.

Wickham, H. (2009). ggplot2: elegant graphics for data analysis. Springer: Nova lorque.

Wickham, H. (2011). The split-apply-combine strategy for data analysis. Journal of Statistical Software, 40(1), 1-29. doi: 10.18637/jss.v040.i01

Winters, K. C., Fawkes, T., Fahnhorst, T., Botzet, A., \& August, G. (2007) A synthesis review of exemplary drug abuse prevention programs in the United States. Journal of Substance Abuse Treatment 32(4), 371-380. doi: 10.1016/j.jsat.2006.10.002 
Érika Pizziolo Monteiro, Mestre em Psicologia pela Universidade Federal de Juiz de Fora (UFJF), é Pesquisadora no Centro de Pesquisa, Intervenção e Avaliação em Álcool e Outras Drogas da Universidade Federal de Juiz de Fora (CREPEIA/UFJF). Endereço para correspondência: Avenida Erico Veríssimo, 830/404. Menino Deus, Porto Alegre/RS. CEP: 90-160180. Telefone: (51) 9527-6194.

E-mail: erikapizziolo@gmail.com

Henrique Pinto Gomide, Mestre em Psicologia pela Universidade Federal de Juiz de Fora (UFJF), é Professor no Centro de Ensino Superior de Juiz de Fora (CES-JF).

E-mail: henriquepgomide@gmail.com

Pollyanna Santos da Silveira, Doutora em Ciências da Saúde pela Universidade Federal de São Paulo (UNIFESP), Pósdoutorado com ênfase em álcool e outras drogas pela Universidade Federal de Juiz de Fora (UFJF), é Professora do Programa de Mestrado em Psicologia da Universidade Católica de Petropólis (UCP) e Pesquisadora do Centro de Pesquisa, Intervenção e Avaliação em Álcool e Outras Drogas da Universidade Federal de Juiz de Fora (CREPEIA/UFJF). E-mail: pollyannassilveira@gmail.com

Telmo Mota Ronzani, Doutor em Ciências da Saúde pela Universidade Federal de São Paulo (UNIFESP), Pós-Doutorado em Saúde Coletiva e Psicologia pela Universidade de São Paulo

(USP), Pós-Doutorado em Saúde Coletiva pela University of Connecticut Health Center (UCHC), é Professor do Departamento de Psicologia e Programa de Pós- Graduação em Psicologia da Universidade Federal de Juiz de Fora (UFJF), Bolsista de Produtividade no Conselho Nacional de Desenvolvimento Científico e Tecnológico (CNPq) e Pesquisador Mineiro na Fundação de Amparo à Pesquisa do Estado de Minas Gerais (FAPEMIG). E-mail: tm.ronzani@gmail.com 